\title{
Uniform Asymptotic Expansion for a Class of Polynomials Biorthogonal on the Unit Circle
}

\author{
N. M. Temme
}

\begin{abstract}
An asymptotic expansion including error bounds is given for polynomials $\left\{P_{n}, Q_{n}\right\}$ that are biorthogonal on the unit circle with respect to the weight function $\left(1-e^{i \theta}\right)^{\alpha+\beta}\left(1-e^{-i \theta}\right)^{\alpha-\beta}$. The asymptotic parameter is $n$; the expansion is uniform with respect to $z$ in compact subsets of $C \backslash\{0\}$. The point $z=1$ is an interesting point, where the asymptotic behavior of the polynomials strongly changes. The approximants in the expansions are confluent hypergeometric functions. The polynomials are special cases of the Gauss hypergeometric functions. In fact, with the results of the paper it follows how (in a uniform way) the confluent hypergeometric function is obtained as the limit of the hypergeometric function ${ }_{2} F_{1}(a, b ; c ; z / b)$, as $b \rightarrow \pm \infty, z \neq b$, with $z=0$ as "transition" point in the uniform expansion.
\end{abstract}

\section{Introduction}

The polynomials considered are

$$
\left\{\begin{aligned}
P_{n}(z ; \alpha, \beta) & ={ }_{2} F_{1}(-n, \alpha+\beta+1 ; 2 \alpha+1 ; 1-z) \\
& =\frac{(\alpha-\beta)_{n}}{(2 \alpha+1)_{n}}{ }_{2} F_{1}(-n, \alpha+\beta+1 ; \beta+1-n-\alpha ; z), \\
Q_{n}(z ; \alpha, \beta) & =P_{n}(z ; \alpha,-\beta) .
\end{aligned}\right.
$$

The biorthogonality means that there is a weight function

$$
\alpha(\theta)=\left(1-e^{i \theta}\right)^{\alpha+\beta}\left(1-e^{-i \theta}\right)^{\alpha-\beta}=(2-2 \cos \theta)^{\alpha}\left(-e^{i \theta}\right)^{\beta},
$$

such that

$$
\begin{array}{rr}
\frac{1}{2 \pi} \int_{-\pi}^{\pi} P_{m}\left(e^{i \theta} ; \alpha, \beta\right) Q_{n}\left(e^{-i \theta} ; \alpha, \beta\right) \alpha(\theta) d \theta=0, & m \neq n, \\
=\frac{\Gamma(2 \alpha+1)}{\Gamma(\alpha+\beta+1) \Gamma(\alpha-\beta+1)} \frac{n !}{(2 \alpha+1)_{n}}, & m=n .
\end{array}
$$

Date received: October 10, 1985. Date revised: June 2, 1986. Communicated by Tom H. Koornwinder. AMS classification: $33 \mathrm{~A} 65,41 \mathrm{~A} 60,33 \mathrm{~A} 30,30 \mathrm{E} 15$.

Key words and phrases: Biorthogonal polynomials, Uniform asymptotic expansion, Heisenberg polynomial, Hypergeometric function. 
A proof of this can be found in [2]. In the same paper the polynomials are considered for large values of $n$, especially for values of $z$ near unity. It is stated that

$$
\lim _{n \rightarrow \infty} P_{n}\left(e^{i \theta / n} ; \alpha, \beta\right)={ }_{1} F_{1}(\alpha+\beta+1 ; 2 \alpha+1 ; i \theta),
$$

which is analogous to a well-known asymptotic result for Jacobi polynomials in terms of Bessel functions. Askey raised the question of how to obtain more terms in the asymptotic result (1.4) and of how to give bounds on the error in the expansion.

In this paper we give the full asymptotic expansion which gives (1.4) as a special case, and we give the error bounds. The result is valid for $z$ ranging in compact subsets of $\mathbf{C} \backslash\{0\}$. So, our expansion especially holds in a neighborhood of $z=1$.

The point $z=1$ is interesting, since $\alpha(\theta)$ vanishes there (that is, $\alpha(\theta)$ vanishes at $\theta=0$, and we write $z=e^{i \theta}$ for $z$ on the unit circle). As Askey remarked, we want to understand the effect of zeros of the weight function on the asymptotic behavior of orthogonal or biorthogonal polynomials. It is also interesting to obtain information on the location of the zeros of $P_{n}$ and $Q_{n}$. A special case gives direct information. Let $\alpha=-\beta=\frac{1}{2}$, then we have

$$
\left\{\begin{array}{l}
P_{n}\left(z ; \frac{1}{2},-\frac{1}{2}\right)=\frac{1}{n+1} \frac{1-z^{n+1}}{1-z}, \\
Q_{n}\left(z ; \frac{1}{2},-\frac{1}{2}\right)=z^{n} .
\end{array}\right.
$$

In this simple case the zeros of $P_{n}$ are uniformly distributed over the unit circle, but those of $Q_{n}$ are all concentrated at $z=0$.

In the theory of special functions, the confluent hypergeometric functions are usually introduced as the limit of the Gauss function:

$$
\lim _{b \rightarrow \infty} F_{1}(a, b ; c ; z / b),
$$

with $a, c$, and $z$ fixed. In some way, this is stated in (1.4). In the definition of the polynomials $P_{n}, Q_{n}$, the parameter $n$ in (1.1) should be entire and nonnegative. However, our asymptotic methods accept real positive values of the large parameter $n$. Since

$$
{ }_{2} F_{1}(a, b ; c ; z)=(1-z)^{c-a-b}{ }_{2} F_{1}(c-a, c-b ; c ; z),
$$

the sign of the large parameter is not relevant.

Consequently, our results can be extended to the more general problem of giving the uniform expansion of

$$
{ }_{2} F_{1}(a, b ; c ; 1-z), \quad a \rightarrow \pm \infty,
$$

$b, c$ fixed and $z$ in compacta of $\mathbf{C} \backslash\{0\}$, and probably for complex values of $a$. This generalization will not be considered here.

We conclude this section with some historical documentation about the biorthogonal system (1.1) (communicated by Tom H. Koornwinder). 
The biorthogonality (1.3) was first stated in [1], while in $[4,(1.15)]$ a proof of (1.3) (different from Askey's proof) was given. It was pointed out there that these polynomials can be expressed in terms of Heisenberg polynomials, which live on the Heisenberg group and were first introduced in [5], while the notation $C_{k}^{(\alpha, \beta)}\left(e^{i \phi}\right)$ introduced in [3] is now standard for these Heisenberg polynomials. Yet another interpretation and proof of the biorthogonality was given in $[6$, see (a) after Remark 3.4]. The present paper uses the notation of [2].'The connection with the Heisenberg polynomials reads

$$
P_{n}\left(e^{i \theta} ; \alpha, \beta\right)=\frac{n !}{(2 \alpha+1)_{n}} e^{i n \theta / 2} C_{n}^{(\alpha-\beta, \alpha+\beta+1)}\left(e^{i \theta / 2}\right) .
$$

\section{The Asymptotic Expansion}

The standard integral for ${ }_{2} F_{1}$-functions gives

$$
P_{n}(z ; \alpha, \beta)=\frac{\Gamma(2 \alpha+1)}{\Gamma(\alpha+\beta+1)} I_{n},
$$

where

$$
I_{n}=\frac{1}{\Gamma(\alpha-\beta)} \int_{0}^{1} t^{\alpha+\beta}(1-t)^{\alpha-\beta-1}(1-t \zeta)^{n} d t, \quad \zeta=1-z .
$$

For convergence of the integral we have the conditions $\alpha+\beta>-1, \alpha-\beta>0$. However, the reciprocal gamma function before the integral removes the singularity due to $\alpha=\beta$. So we assume that

$$
\alpha+\beta>-1 \text {. }
$$

When complex values of $\alpha, \beta$ are used, the condition is $\operatorname{Re}(\alpha+\beta)>-1$. Put

$$
1-t \zeta=z^{u}=e^{u \ln z},
$$

with $\ln z$ the principal branch of the logarithm, which is real when $z>0$. Then we have

$$
\left\{\begin{aligned}
I_{n} & =z^{\alpha-\beta-1}\left[\frac{\ln z}{z-1}\right]^{2 \alpha} J_{n}, \\
J_{n} & =\frac{1}{\Gamma(\alpha-\beta)} \int_{0}^{1} f(u) u^{\alpha+\beta}(1-u)^{\alpha-\beta-1} e^{\omega u} d u \\
f(u) & =\left[\frac{1-z^{u}}{-u \ln z}\right]^{\alpha+\beta}\left[\frac{1-z^{u-1}}{(1-u) \ln z}\right]^{\alpha-\beta-1}, \\
\omega & =(n+1) \ln z .
\end{aligned}\right.
$$

The function $f$ is holomorphic in a neighborhood of $[0,1]$; singularities occur at

$$
u_{k}=\frac{2 k \pi i}{\ln z}, \quad v_{m}=1+\frac{2 m \pi i}{\ln z}, \quad k, m \in \mathbf{Z} \backslash\{0\} .
$$


So when $z$ ranges through compact subsets of $\mathbf{C} \backslash\{0\}$, the singularities of $f$ are bounded away from $[0,1]$.

Before constructing the uniform asymptotic expansion we first remark that simpler, i.e., nonuniform expansions can be obtained for two separate cases:

(i) $\operatorname{Re} \omega=(n+1) \ln |z|<0, z$ fixed.

The dominant point in the integral $J_{n}$ is $u=0$, and the asymptotic expansion follows by expanding the function $f(u)$ at $u=0$. The result is

$$
P_{n}(z ; \alpha, \beta) \sim \frac{\Gamma(2 \alpha+1)}{\Gamma(\alpha-\beta)}[(n+1)(1-z)]^{-\alpha-\beta-1}, \quad \text { as } \quad n \rightarrow \infty .
$$

(ii) $\operatorname{Re} \omega>0, z$ fixed.

Now the dominant point is $u=1$ and an expansion of $f(u)$ at $u=1$ has to be used. In this case

$$
P_{n}(z ; \alpha, \beta) \sim \frac{\Gamma(2 \alpha+1)}{\Gamma(\alpha+\beta+1)} z^{n+\alpha-\beta}(z-1)^{\beta-\alpha}(n+1)^{\beta-\alpha}, \quad \text { as } \quad n \rightarrow \infty .
$$

In the uniform expansion, contributions from both $u=0$ and $u=1$ will be taken into account. In this way we can allow $\operatorname{Re} \omega$ to be negative as well as positive; even $\omega=0$ is accepted.

Observe that $\operatorname{Re} \omega>0, \operatorname{Re} \omega<0$ is equivalent to $|z|>1,|z|<1$, respectively, so that in fact all points on the unit circle in the $z$-plane are "transition" points; i.e., points for which the asymptotic behavior of the polynomials $P_{n}, Q_{n}$ will change drastically. For polynomials this is not surprising, of course. However, in (2.2), (2.6), (2.7) and in the following analysis $n$ need not be an integer.

A uniform expansion for $J_{n}$ of (2.4) is obtained as follows. We write

$$
f(u)=\alpha_{0}+\beta_{0} u+u(1-u) g_{0}(u)
$$

with $\alpha_{0}=f(0), \beta_{0}=f(1)-f(0)$. Then $J_{n}$ of (2.4) equals

$$
J_{n}=\alpha_{0} \varphi_{0}+\beta_{0} \varphi_{1}+H_{n},
$$

with

$$
\left\{\begin{array}{l}
\varphi_{0}=\frac{\Gamma(\alpha+\beta+1)}{\Gamma(2 \alpha+1)}{ }_{1} F_{1}(\alpha+\beta+1 ; 2 \alpha+1 ; \omega), \\
\varphi_{1}=\frac{\Gamma(\alpha+\beta+2)}{\Gamma(2 \alpha+2)}{ }_{1} F_{1}(\alpha+\beta+2 ; 2 \alpha+2 ; \omega)=\frac{d}{d \omega} \varphi_{0} .
\end{array}\right.
$$

Integration by parts gives for $H_{n}$, when $\omega \neq 0$,

$$
\begin{aligned}
H_{n} & =\frac{1}{\omega \Gamma(\alpha-\beta)} \int_{0}^{1} g_{0}(u) u^{\alpha+\beta+1}(1-u)^{\alpha-\beta} d e^{\omega u} \\
& =\frac{1}{\omega \Gamma(\alpha-\beta)} \int_{0}^{1} f_{1}(u) u^{\alpha+\beta}(1-u)^{\alpha-\beta-1} e^{\omega u} d u,
\end{aligned}
$$


with

$$
f_{1}(u)=[(2 \alpha+1) u-\alpha-\beta-1] g_{0}(u)-u(1-u) g_{0}^{\prime}(u) .
$$

This new $f_{1}$ has the same domain of regularity as $g_{0}$ and $f$. By repeating the above procedure, we obtain the formal expansion

$$
J_{n} \sim \varphi_{0} \sum_{m=0}^{\infty} \frac{\alpha_{m}}{\omega^{m}}+\varphi_{1} \sum_{m=0}^{\infty} \frac{\beta_{m}}{\omega^{m}}
$$

with

$$
\left\{\begin{aligned}
\alpha_{m} & =f_{m}(0), \quad \beta_{m}=f_{m}(1)-f_{m}(0), \quad f_{0}=f \\
f_{m}(u) & =[(2 \alpha+1) u-\alpha-\beta-1] g_{m-1}(u)-u(1-u) g_{m-1}^{\prime}(u) \quad(m \geq 1) \\
& =\alpha_{m}+\beta_{m} u+u(1-u) g_{m}(u) \quad(m \geq 0)
\end{aligned}\right.
$$

and where $\omega=(n+1) \ln z$.

The restrictions on $\alpha, \beta$ are as in (2.3). For $\omega$ we temporarily suppose $\omega \neq 0$. In the following section we prove that

$$
A_{m}=\frac{\alpha_{m}}{\ln ^{m} z}, \quad B_{m}=\frac{\beta_{m}}{\ln ^{m} z}, \quad m=0,1, \ldots,
$$

are regular functions of $z$ in $C \backslash\{0\}$. So, the complete expansion for $P_{n}$ is

$$
\begin{aligned}
P_{n}(z ; \alpha, \beta) \sim & \frac{\Gamma(2 \alpha+1)}{\Gamma(\alpha+\beta+1)} z^{\alpha-\beta-1}\left[\frac{\ln z}{z-1}\right]^{2 \alpha} \\
& \times\left[\varphi_{0} \sum_{m=0}^{\infty} \frac{A_{m}}{(n+1)^{m}}+\varphi_{1} \sum_{m=0}^{\infty} \frac{B_{m}}{(n+1)^{m}}\right],
\end{aligned}
$$

as $n \rightarrow \infty$; the expansion holds uniformly with respect to $z$ in compact subsets of $C \backslash\{0\}$. The same holds for $Q_{n}(z ; \alpha, \beta)$ with $\beta$ replaced by $-\beta$ (also in (2.3)). So we have for both $P_{n}$ and $Q_{n}$ an expansion as in (2.14) when $-\alpha-1<\beta<\alpha+1$; when $\alpha$ and $\beta$ are complex, the real parts of $\alpha, \beta$ should satisfy these inequalities.

The expansion (2.14) contains (1.4) as a special case, since for $z=1$ we have $\alpha_{0}=1, \beta_{0}=0$.

An error bound for the expansion in (2.14) follows easily from the integration by parts procedure. Writing for $J_{n}$ of (2.4)

$$
J_{n}=\varphi_{0} \sum_{m=0}^{k-1} \frac{A_{m}}{(n+1)^{m}}+\varphi_{1} \sum_{m=0}^{k-1} \frac{B_{m}}{(n+1)^{m}}+R_{k}, \quad k=0,1, \ldots,
$$

we have for $R_{k}$ the representation

$$
R_{k}=\frac{1}{\omega^{k} \Gamma(\alpha-\beta)} \int_{0}^{1} f_{k}(u) u^{\alpha+\beta}(1-u)^{\alpha-\beta-1} e^{\omega u} d u .
$$

Again, $f_{k}(u) / \ln ^{k} z$ is regular (see the next section) and we define positive numbers $M_{k}$ not depending on $u$ such that

$$
\left|f_{k}(u)\right| \leq\left|\ln ^{k} z\right| M_{k}, \quad u \in[0,1], \quad z \text { in a compact subset of } \mathbf{C} \backslash\{0\} .
$$


Then we obtain

$$
\left|R_{k}\right| \leq \frac{1}{(n+1)^{k}} M_{k}\left|\bar{\varphi}_{0}\right|
$$

where $\bar{\varphi}_{0}$ is $\varphi_{0}$ of (2.9) with $\omega$ replaced by $\operatorname{Re} \omega$. This gives an error bound for the asymptotic expansion and it shows the asymptotic nature of (2.14).

\section{On the Regularity of $A_{m}, B_{m}$ at $z=1$}

In this section we show that the coefficients of (2.14) defined in (2.13) are regular functions of $z$, especially when $\lambda:=\ln z=0$. We also show that $\left|f_{k}(u)\right|$ can be bounded as in (2.17), again when $\lambda=0$. We suppose in this section that $|\lambda|$ is small, say $|\lambda| \leq \lambda_{0}$, where $\lambda_{0}$ is a fixed small positive number.

Before proving the regularity of $A_{m}$ and $B_{m}$ we remark that $f(u)$ of (2.4) depends in a crucial way on the uniformity parameter $\ln z$. The result (2.14) is certainly not true for more general functions, say functions just regular on a set in the complex $u$-plane containing $[0,1]$ in its interior.

Inspection of $f$ of (2.4) shows that it can be written as

$$
f(u)=\varphi(\lambda u) \psi(\lambda(u-1)), \quad \lambda=\ln z,
$$

where

$$
\varphi(x)=\left(\frac{e^{x}-1}{x}\right)^{\alpha+\beta}, \quad \psi(x)=\left(\frac{e^{x}-1}{x}\right)^{\alpha-\beta-1} .
$$

The fact that in both $\varphi(x)$ and $\psi(x)$ the same function of $x$ appears is not so important. However, it is crucial that the parameter $\lambda$ appears in both $\varphi$ and $\psi$ in (3.1). We expand $f(u)$ in powers of $\lambda$. That is, we write

$$
f(u)=\sum_{m=0}^{\infty} p_{m} \lambda^{m} u^{m} \sum_{m=0}^{\infty} q_{m} \lambda^{m}(u-1)^{m}=\sum_{m=0}^{\infty} r_{m}(u) \lambda^{m},
$$

where

$$
r_{m}(u)=\sum_{k=0}^{m} p_{k} q_{m-k} u^{k}(u-1)^{m-k}
$$

Observe that $r_{m}$ is a polynomial in $u$ of degree $m$. Since $|\lambda|$ is small, the manipulations of the series in (3.3) holds for $u$-values in a set $U$ in the $u$-plane that contains $[0,1]$ in its interior; the $r_{m}$-series in (3.3) converges uniformly with respect to $u \in U$.

By using (3.3) and (2.8) we infer that $g_{0}$ can be written in the form

$$
g_{0}(u)=\sum_{m=0}^{\infty} s_{m}(u) \lambda^{m}, \quad \text { with } \quad s_{0}=s_{1}=0,
$$

where $s_{m}$ is a polynomial in $u$ of degree $m-2(m \geq 2)$. 
The proof of this is easily established by using a Cauchy integral representation for $g_{0}$. From (2.8) it follows that

$$
g_{0}(u)=\frac{1}{2 \pi i} \int_{C} \frac{f(v)}{v(1-v)(v-u)} d v,
$$

where $C$ is a contour in the above-mentioned domain $U ; C$ encloses the interval $[0,1]$. This representation follows by writing $f(u), f(1), f(0)$ as similar contour integrals. Substituting the $r_{m}$-series of (3.3) we obtain for $s_{m}$ in (3.4)

$$
s_{m}(u)=\frac{1}{2 \pi i} \int_{C} \frac{r_{m}(v)}{v(1-v)(v-u)} d v .
$$

Since $r_{m}(v)$ is a polynomial of degree $m, s_{0}=s_{1}=0$, which establishes (3.4); it is also clear that $s_{m}(u)$ is a polynomial of degree $m-2$ for $m \geq 2$.

We next consider $f_{1}$ of (2.10). Writing

$$
f_{1}(u)=\sum_{m=0}^{\infty} r_{m}^{(1)}(u) \lambda^{m}
$$

we obtain

$$
\left.r_{m}^{(1)}(u)=[(2 \alpha+1) u-\alpha-\beta-1] s_{m}(u)-u(1-u) s_{m}^{\prime}(u)\right] .
$$

So, $r_{m}^{(1)}$ is a polynomial of degree $m-1, r_{0}^{(1)}=r_{1}^{(1)}=0$. This shows that $f_{1}(u) / \lambda$ is regular for all $u \in U$ and $|\lambda|<\lambda_{0}$; so $A_{1}, B_{1}$ are regular for $|\lambda|<\lambda_{0}$. The same procedure can be used for the higher coefficients $A_{k}, B_{k}$ in (2.14) and to establish the meaning of (2.17) for $z \rightarrow 1$ or $\lambda \rightarrow 0$, for all $k \geq 0$.

As remarked earlier, the special form of $\varphi$ and $\psi$ in (3.1) is not important. In fact, the method applies to more general functions $f$, or $\varphi$ and $\psi$, as long as the representation (3.1) remains and $\varphi$ and $\psi$ are regular in some neighborhood of $[0,1]$. Use of analytic functions and of Cauchy integrals is not needed. The verification of the regularity of $f_{k}(u) / \ln ^{k} z$ as $z \rightarrow 1$ can be proved without using (3.5), but the present proof is rather elegant and short.

\section{Concluding Remarks}

(i) Although the coefficients $\alpha_{m}, \beta_{m}$ in (2.11) are defined in terms of a recursion relation (2.12), the evaluation of these coefficients, and hence of $A_{m}, B_{m}$ in (2.12), is a tedious process. Especially, the evaluation of $A_{m}, B_{m}$ for $z$ at or near unity is difficult. A completely different approach to obtain (2.14) can be based on the differential equation of the Gauss functions. By substituting (2.14) into a transformed version of this equation a recursive system is obtained for $A_{m}, B_{m}$ and their derivatives. Taylor expansions of $A_{m}, B_{m}$ around $z=1$ can then be substituted to compute coefficients of these expansions. In Olver [7] asymptotic methods for special functions are usually based on differential equations.

(ii) In the discussion of (2.6), (2.7) we observed that the role of the critical points $u=0, u=1$ of $J_{n}$ in (2.4) is interchanged when $\log |z|$ changes sign. The 
confluent hypergeometric functions $\varphi_{0}, \varphi_{1}$ of (2.9) are exponentially large when $\operatorname{Re} \omega \rightarrow+\infty$. When $\operatorname{Re} \omega \rightarrow-\infty$ they are of algebraic growth in $\omega$, and not exponentially small. These asymptotic features and the use of confluent hypergeometric functions as approximants in such problems have not been discussed in the earlier asymptotic literature, as far as I know.

(iii) The special case $2(\alpha+\beta+1)=2 \alpha+1$ (or $\beta=-\frac{1}{2}$ ) makes a Bessel function of $\varphi_{0}$ in (2.9). It also gives the integrand of $J_{n}$ in (2.4) some symmetry. In this form the asymptotic problem resembles that of certain Legendre functions, as considered in Ursell [10]. The simple case $\alpha=\frac{1}{2}, \beta=\frac{1}{2}$ yields for $\varphi_{0}$ the spherical Bessel function of order zero (see (1.5)). Furthermore, in the case $\beta=-\frac{1}{2}$ not only the confluent hypergeometric function becomes a Bessel function, but also

$$
P_{n}\left(e^{i \theta} ; \alpha,-\frac{1}{2}\right)=\frac{n !}{(2 \alpha+1)_{n}} e^{i n \theta / 2} C_{n}^{\alpha+1 / 2}\left(\cos \frac{1}{2} \theta\right), \quad \text { a Gegenbauer polynomial. }
$$

Thus the familiar asymptotics of Gegenbauer polynomials in terms of Bessel functions must in fact be a special case of the results in this paper.

(iv) A similar asymptotic has been considered in the literature for

$$
{ }_{2} F_{1}\left(\frac{1}{2}(i \lambda+\alpha+\beta+1), \frac{1}{2}(-i \lambda+\alpha+\beta+1) ; \alpha+1 ;-\sinh ^{2}(t / \lambda)\right)
$$

as $\lambda$ tends to $\infty$, cf. [8] and [9].

Acknowledgment. The author thanks the referees and Tom Koornwinder (editor) for their valuable comments on an earlier version of the paper.

\section{References}

1. R. Askey (1982): Discussion of Szegö's paper "Beiträge zur Theorie der Toeplitzschen Formen". In: G. Szegö, Collected Works, Vol. I (R. Askey, ed.). Boston: Birkhäuser, pp. 303-305.

2. R. Askey (1985): Some problems about special functions and computations. In: Proceedings of the Conference on Special Functions: Theory and Computation (October 1984, Torino). Torino: Rendiconti del Seminario Matematico.

3. G. Gasper (1981): Orthogonality of certain functions with respect to complex valued weights. Canad. J. Math., 33:1261-1270.

4. P. C. Greiner, T. H. Koornwinder (1983): Variations on the Heisenberg spherical harmonics, Report ZW 186/83. Amsterdam: Mathematisch Centrum.

5. P. C. Greiner (1980): Spherical harmonics on the Heisenberg group. Canad. Math. Bull., 23:383396.

6. E. Hendriksen, H. van Rossum (1986): Orthogonal Laurent polynomials. Nederl, Akad. Wetensch. Indag. Math., 48:17-36.

7. F. W. J. Olver (1974): Asymptotics and Special Functions. New York: Academic Press.

8. R. J. Stanton, P. A. Tomas (1978): Expansions for spherical functions on noncompact symmetric spaces. Acta Math., 140:251-271.

9. K. Trimèche (1981): Transformation integrale de Weyl et theórème de Paley-Wiener associés à un opérateur différentiel singulier sur $(0, \infty)$. J. Math. Pures Appl., 9, 60:51-98.

10. F. Ursell (1984): Integrals with a large parameter: Legendre functions of large degree and fixed order. Math. Proc. Cambridge Philos. Soc., 95:367-380.

N. M. Temme

Centre for Mathematics and Computer Science

P.O. Box 4079

1009 AB Amsterdam

The Netherlands 